\title{
Prospective Motion Correction and Automatic Segmentation of Penetrating Arteries in Phase Contrast MRI at 7 T
}

Julia Moore, ${ }^{1,2}$ Jordan Jimenez, ${ }^{1}$, Weili Lin, ${ }^{1,3}$ William Powers ${ }^{4}$ Xiaopeng Zong, ${ }^{1,3, *}$

1. Biomedical Research Imaging Center, 2. Department of Biology, 3. Department of Radiology, 4. Department of Neurology, University of North Carolina at Chapel Hill, Chapel Hill, NC, USA

*Corresponding Author: Xiaopeng Zong, Department of Radiology and Biomedical Research Imaging Center, University of North Carolina at Chapel Hill, CB\#7515 Chapel Hill, NC, 27599, Email: xiaopeng_zong@med.unc.edu, Phone: 515-450-7196

\section{Running head}

Prospective Motion Correction and Deep-learning Based Segmentation of Penetrating Arteries in Centrum Semiovale in Phase Contrast MRI at $7 \mathrm{~T}$

Word count: 5000 


\begin{abstract}
Purpose: To develop a prospective motion correction (MC) method for phase contrast (PC) MRI of penetrating arteries (PA) in centrum semiovale at $7 \mathrm{~T}$ and evaluate its performance using automatic PA segmentation.
\end{abstract}

Methods: Head motion was monitored and corrected during the scan based on fat navigator images. Two convolutional neural networks $(\mathrm{CNN})$ were developed to automatically segment PAs and exclude surface vessels. Real-life scans with MC and without MC (NoMC) were performed to evaluate the MC performance. Motion score was calculated from the range of translational and rotational motion parameters. MC vs NoMC pairs were divided according to their score differences into groups with similar, less, or more motions during MC. Data reacquisition was also performed to evaluate whether it can further improve PA visualization.

Results: In the group with similar motion, more PA counts $\left(\mathrm{N}_{\mathrm{PA}}\right)$ were obtained with $\mathrm{MC}$ in 9 $(60 \%)$ cases, significantly more than the number of cases (1) with less PAs ( $p=0.011$; binomial test). In the group with less motion during $\mathrm{MC}, \mathrm{MC}$ images had more or similar $\mathrm{N}_{\mathrm{PA}}$ in all cases, while in the group with more motion during $\mathrm{MC}$, the numbers of cases with less and more $\mathrm{N}_{\mathrm{PA}}$ during MC were not significantly different (3 vs 0 ). Data reacquisition did not further increase $\mathrm{N}_{\mathrm{PA}}$. CNNs had higher sensitivity (0.85) and accuracy (Dice coefficient 0.85 ) of detecting PAs than a threshold based method.

Conclusions: Prospective $\mathrm{MC}$ and $\mathrm{CNN}$ based segmentation improved the visualization and delineation of PAs in PC MRI at $7 \mathrm{~T}$.

\title{
KEYWORDS
}

Prospective motion correction, phase contrast MRI, convolutional neural network, image segmentation, penetrating arteries 


\section{INTRODUCTION}

Cerebral small vessel disease (SVD) is a condition postulated to have multiple different etiologies. It may result from a cascade of events, starting with endothelial dysfunction in the penetrating arteries (PAs) [1]. Other causes include the thickening of the arterial media due to lipohyalinosis or obstruction of the origins of PAs by parent artery intimal plaques, which lead to brain ischemia resulting in deep small infarcts and leakage of fluid causing edema and later gliosis in white matter tracts [2]. Additionally, inflammation might also be involved in the development of PA leakage and endothelial dysfunction, although the causal relationships are yet to be established [3]. Cumulatively, SVD might lead to substantial cognitive [4-6], psychiatric[7], and physical disabilities [8, 9].

Phase contrast (PC) MRI is a common method for imaging arteries and can visualize a large number of PAs $(27$ - 91) in centrum semiovale at $7 \mathrm{~T}[10,11]$. One major concern that arises during imaging is the susceptibility to motion which reduces PA visibility and might introduce artificial group differences due to different motion tendencies between the participants. As the PC MRI only covers a single slice, prospective motion correction (MC) is needed to ensure consistent imaging location in the presence of head motion. In this study, we will evaluate the improvements of PA visualization by prospective MC based on motion parameters derived from whole-brain fat navigator (FatNav) images [12].

Accurate segmentation of penetrating arteries is a key step in objective evaluation of improved $\mathrm{PA}$ visualization by MC and in future studies delineating the roles of PAs in small vessel disease. The existing segmentation method relied on thresholding of the phase and magnitude image intensities which only controls for false positives rate, but might suffer from a high false negative rate due to the low contrast to noise ratio of some PAs in the images $[10,11]$. Here, we evaluate the performance of a two-dimensional multi-channel multi-scale encoder decoder network (M2EDN) for segmenting the PAs, which was adopted from a three-dimensional M2EDN

originally developed for perivascular spaces segmentation [13]. Furthermore, since some arteries on cortical surface mimic penetrating arteries, a white matter (WM) mask can be applied to remove such false PAs and further improve segmentation accuracy. To this end, we also developed a 3D U-NET based method to obtain the WM mask, utilizing 3D T2-weighted (T2w) images that were acquired during the same imaging session [14]. 


\section{METHODS}

\subsection{Subjects}

This study was approved by the Institutional Review Board of the University of North Carolina at Chapel Hill. Written informed consents were obtained from all subjects before the scan. Two separate experiments were performed: Experiment 1 (Exp 1) acquired images used for training the convolutional neural networks $(\mathrm{CNN})$ parameters and Experiment $2(\operatorname{Exp} 2)$ were carried out to evaluate the performance of MC. 39 healthy volunteers (aged 21 - 55 years, 28 females) were included in Exp 1 and 21 patients with diabetes and 13 healthy controls (aged $37-70$ years, 19 females) were included in $\operatorname{Exp} 2$.

\subsection{Data acquisition}

All images were acquired on a 7T MRI scanner (Siemens Healthineer, Erlangen, Germany). In Exp 1, a Nova 32-channel receiver and 8-channel transmitter head coil (Nova Medical, Wilmington, MA, USA) was used. No radio frequency magnetic field $\left(\mathrm{B}_{1}\right)$ shimming was performed. In Exp 2, the images were acquired using a Nova 32-channel receiver and singlechannel volume transmitter coil. The subjects were asked to keep their heads still during all scans.

A 3D variable flip angle turbo spin echo (TSE) sequence was used to acquire T2w images for WM segmentation $[15,16]$. A FatNav image [12] was acquired within each repetition time (TR) to monitor head motion. In Exp 1, the FatNav images were not utilized to correct motion in the present study, although they had been utilized to perform retrospective motion correction in a separate study [17]. In Exp 2, the FatNav images were used to estimate motion parameters relative to the first FatNav image which were then used to adjust the imaging FOV for the next TR.

Then, a single slice PC MRI was performed to image PAs in centrum semiovale. The slice was positioned $15 \mathrm{~mm}$ above the corpus callosum and parallel to the anterior commissure - posterior commissure line. No FatNav was acquired for the PC MRI scans in Exp 1. In Exp 2, the FatNav and PC MRI data were acquired alternatively as shown in the sequence diagram in Fig. 1. After acquiring and reconstructing each FatNav, motion parameters were estimated using vendor provided module (MOCO functor) and the PC MRI slice position and readout, phase encoding, 
and slice normal directions were adjusted accordingly. As the slice was prescribed based on the TSE images, to correct for potential motion between the TSE and PC MRI scans, the FatNavs in PC MRI were registered to the first FatNav from the TSE-MRI scan.

Because it took $4.68 \mathrm{~s}$ to acquire a complete FatNav in PC MRI and an additional $2.1 \mathrm{~s}$ to reconstruct the image, motion occurred due this period cannot be corrected. Therefore, reacquiring k-space data during periods with large motion may further improve image quality. For this purpose, $\mathrm{k}$ space data were divided by the times when the motion parameter feedbacks were received into blocks. Denote the motion parameters received at the beginning and end of data block $i\left(D_{\mathrm{i}}\right)$ as $M_{\mathrm{i}-1}$ and $M_{\mathrm{i}}$, corresponding FatNav images as $F_{\mathrm{i}-1}$ and $F_{\mathrm{i}}$, and the relative translation and rotation between $F_{\mathrm{i}-1}$ and $F_{\mathrm{i}}$ as $T_{\mathrm{i}}$ and $R_{\mathrm{i}}$ which can be calculated from $M_{\mathrm{i}-1}$ and $M_{\mathrm{i}} . D_{\mathrm{i}}$ was reacquired if $T_{\mathrm{i}-1}, T_{\mathrm{i}}$, or $T_{\mathrm{i}+1}$ was greater than $0.1 \mathrm{~mm}$ or $R_{\mathrm{i}-1}, R_{\mathrm{i}}$, or $R_{\mathrm{i}+1}$ was greater than $0.1^{\circ}$. The requirements ensured that the motion was below the specified thresholds during the $F_{\mathrm{i}-1}, F_{\mathrm{i}}$, and $D_{\mathrm{i}}$ acquisition periods. The reacquired data was in the same temporal order as the original data. After the completion of data reacquisition, the same criterion was used to determine whether the required data needed to be reacquired one more time. The scan stopped until no further reacquisition was needed or the total reacquisition time exceeded the original scan time.

Three $(\mathrm{n}=32)$ or two $(\mathrm{n}=1)$ PC MRI scans with MC were performed in each subject. Data reacquisition was carried out in all scans in 18 subjects but only in the last scan in 15 subjects. For comparison, a fourth scan without MC (NoMC) was performed in 14 subjects where the slice position was not updated while the FatNavs were still acquired for monitoring motion. Furthermore, to study whether the addition of the FatNav images would impact the quality of PC MRI images, the PC MRI with MC and the conventional PC MRI scan without the FatNav module were acquired in an additional subject, while the subject kept the head still.

At the beginning of each sequence that contained FatNav, a navigator image that included fully sampled GRAPPA calibration data at the k-space center was acquired. The parameters for all the sequences are provided in Supporting Information Table S1.

\subsection{Segmentation}

\section{M2EDN for PA segmentation}


The network architecture for PA segmentation is the same as that proposed in [13] except for two modifications. First, the phase and magnitude images were used as network input channels instead of the original and preprocessed images. Second, the convolution and pooling layers were changed from 3-dimensional to 2-dimensional since the input images were twodimensional. In addition, due to the smaller memory size required for 2D images, the entire images were fed into the network without separating them into patches.

\section{U-NET for WM segmentation}

The network consists of 4 steps in the contracting path and another 4 steps in the expansive path. The contracting path applies two $3 \times 3 \times 3$ convolutional layers in each step. In between these convolutional layers, we incorporated a dropout layer of $0.1,0.2$, or 0.3 . And specific to the contracting path, different spatial scales were connected by $2 \times 2 \times 2$ max-pooling layers with a stride of 2 [18]. We started with an initial patch size of 128x128x128 and 16 channels. Channel number doubled with each step in the contracting path $(32,64,128,256)$ but patch size was halved with each step $(64 \times 64 \times 64,32 \times 32 \times 32,16 \times 16 \times 16,8 \times 8 \times 8 \ldots)$. The same pattern exists in the expansive path back to 16 channels and $128 \times 128 \times 128$ patch size. The expansive path upsamples the image back to its original size using transposed convolutions. Once the image is upsampled, it is combined with the image at the same spatial scale from the contracting path to get a more accurate result. Lastly, a 1x1 convolutional layer is applied to produce the output with a 5 channels, which is followed by a softmax layer to produce the probabilities of the background plus four tissue types, including thalamus, basal ganglia, WM, and midbrain, the same ROIs as in our previous study $[19,20]$. The architecture of the U-NET can be found in Supporting Information Fig. S1.

\section{$\underline{\text { Ground truth }}$}

Ground truth (GT) masks for training the networks were manually drawn in ITK-Snap and custom made Matlab (MathWorks, Natick, MA, USA) programs. The four tissue ROIs were defined in 10 TSE images from Exp 1. PAs masks were defined as hyperintensive voxels in the phase image inside WM on 61 PC MRI images from Exp 1. To control for false positive voxels, each hyperintensive cluster had to overlap with a hyperintensive cluster on the corresponding magnitude image. 


\section{Training and testing}

Both networks were implemented using Keras and trained using the ground truth masks defined above. The image - GT mask pairs were divided into 10 groups. Nine groups were selected for training and the remaining one for testing the model performance. To enhance the training dataset, new training data were generated by the following procedures: 1) flip the T2w image GT mask pairs in the L-R direction, 2) rotate the pairs by 10 and -10 degrees around the I-S and L-R axes. 3) flip the PC MRI image - GT mask pairs in the L-R direction. The training was performed for 40 Epochs with batch sizes of 4 and 2 for U-NET and M2EDN. Convergence of the training was confirmed by plotting the sensitivity (SEN), positive predictive value (PPV), and dice similarity coefficient (DSC) versus the number of epochs. For U-NET, the Adam optimizer was employed with a learning rate of 0.0001 and categorical cross entropy loss function For M2EDN, the same optimizer was employed but with a learning rate of 0.001 and the loss function was the same as defined in [13].

Using the group that were left out for testing, we numerically analyzed segmentation performances of our networks using SEN, PPV, and DSC as defined below:

$$
\begin{gathered}
\mathrm{PPV}=\mathrm{TP} /(\mathrm{TP}+\mathrm{FP}) \\
\mathrm{SEN}=\mathrm{TP} /(\mathrm{TP}+\mathrm{FN}) \\
\mathrm{DSC}=2 \mathrm{TP} /(2 \mathrm{TP}+\mathrm{FP}+\mathrm{FN}),
\end{gathered}
$$

where TP, FP, and FN stand for the number of true positive, false positive, and false negative voxels, respectively, using the manually drawn masks as ground truth. Additionally, since the true size of PAs are less than 1 voxel in the PC MRI images and the main goal of the segmentation algorithm is to simply detect visible PAs, but not to delineate their spatial extent. the three parameters are also calculated using TP, FP, FN for true positive, false positive and false negative spatially connected clusters. A predicted cluster is considered to be a true cluster if it spatially overlaps with a cluster in the ground truth mask. For PA segmentation, the three parameters were calculated both before and after applying WM masks to removed FP voxels.

\section{Threshold based PA segmentation}

For comparison, we also obtained PA masks using a threshold-based method as described in [10]. Briefly, voxels with signal intensity two standard deviations above the smoothed 
neighboring intensity were identified in the phase images and the magnitude images with flow encoding gradient off. The PA mask was then defined as clusters in the phase image that overlapped with a cluster in the magnitude image within WM. Clusters within regions with ghosting artifacts due to large surface vessels were manually excluded.

\subsection{Data analysis}

\subsubsection{Image reconstruction.}

The FatNav images were reconstructed using the standard GRAPPA reconstruction algorithm after inverse Fourier transform (IFT) of the data along the readout direction [21]. A separate kernel with a size of $2 \times 2$ was estimated for each position along the readout to calculate the missing k-space data. The missing data due to partial Fourier acquisition was zero filled before IFT along PAR and PE directions. The images from all channels were combined into a single image by root mean square. The TSE images were reconstructed by the vendor provided software on the scanner.

PC MRI images were reconstructed offline to a voxel size of $0.1563 \times 0.1563 \mathrm{~mm}^{2}$ by zero padding in k-space before inverse Fourier transform. Images from repetitions 2 - 10 were averaged. The first repetition was discarded because there was inherent slice position inconsistency between $\mathrm{k}$-space data received before and after receiving the first feedback during the first repetition. The inconsistency was caused by subtle differences between the reference FatNav images acquired during the TSE and PC MRI sequences, which caused systematic errors (nonzero biases) in estimated motion parameters. Then, coil-combined phase and magnitude images were obtained as described in [10].

To study whether replacing data affected by motion can further improve image quality, we further replaced data block $D_{\mathrm{i}}$ in the initial acquisition by a reacquired block $D_{\mathrm{j}}$ at the same kspace locations if the sum of the maximums of $T_{\mathrm{i}-1}, T_{\mathrm{i}}$, and $T_{\mathrm{i}+1}\left(\operatorname{Tmax}_{\mathrm{i}}\right)$ and of $R_{\mathrm{i}-1}, R_{\mathrm{i}}$, and $R_{\mathrm{i}+1}$ $\left(\operatorname{Rmax}_{\mathrm{i}}\right)$ was greater than $\operatorname{Tmax}_{\mathrm{j}}+\mathrm{Rmax}$, before the above image reconstruction.

\subsubsection{PA segmentation}

Out of the ten trained models for each network, the one with the largest DSC (DSC of WM in the case of U-NET) was selected to segment the PC MRI and TSE images in Exp 2. To generate the WM masks, we first resampled the WM masks generated by U-NET at the pixel positions of the 
PC MRI images. Then, the resampled WM masks were manually adjusted to account for possible head movement between the T2w and PC MRI scans (Exp 1) or for above-mentioned systematic error in FatNav image registration (Exp 2). In Exp 1, the WM masks were only available for 50 of the 61 PC MRI images. The number of PAs $\left(\mathrm{N}_{\mathrm{PA}}\right)$ is defined as the number of spatially connected clusters in the final masks.

\subsubsection{Motion parameters}

To investigate whether the $\mathrm{N}_{\mathrm{PA}}$ difference between $\mathrm{MC}$ and NoMC scans depended on the motion severity, rotational $\left(M_{\mathrm{R}}\right)$ and translational $\left(\mathrm{M}_{\mathrm{T}}\right)$ motion ranges were calculated for all scans. $M_{\mathrm{R}}\left(M_{\mathrm{T}}\right)$ was defined as the root mean square of the differences of the maximum and minimum values of the three rigid-body rotational (translational) parameters of all FatNav images acquired during repetitions $2-10$.

\subsubsection{Statistical Analysis}

Wilcoxon's signed rank tests were performed to compare the PPV, SEN, and DSC values of the various PA segmentation methods.

To evaluate whether MC improves PA visualization, the $\mathrm{MC}$ and NoMC image pairs were formed by pairing the NoMC and each of the MC scans from the same subject. The image pairs were visually inspected and those showing clear spatial mismatch (likely due to motion during or before the NoMC scans) between the two images were excluded. As a result, images from 5 out of the 14 subjects were excluded, resulting in 26 pairs for comparison. The pairs were divided into three groups based on their difference in motion parameters. A motion score was defined as: score $=M_{\mathrm{T}}+57.3 \mathrm{~mm} \times M_{\mathrm{R}}$, where $\mathrm{M}_{\mathrm{T}}$ and $\mathrm{M}_{\mathrm{R}}$ are in units of $\mathrm{mm}$ and radians, respectively. The motion score is similar to that defined in [22] and corresponds to adding to $M_{\mathrm{T}}$ a shift caused by rotation $M_{\mathrm{R}}$ of a point on the surface of a sphere with a radius of $57.3 \mathrm{~mm}$. For simplicity, the radius of $57.3 \mathrm{~mm}$ is chosen instead of the $64 \mathrm{~mm}$ in [22] such that the score is simply equal to the sum of $M_{\mathrm{T}}$ and $M_{\mathrm{R}}$ when they are expressed in mm and degrees, respectively. The pairs with motion score difference between $\mathrm{MC}$ and NoMC in the ranges of $\left(-\infty,-\theta_{\mathrm{M}}\right),\left[-\theta_{\mathrm{M}}\right.$, $\theta_{\mathrm{M}} \mathrm{]}$, and $\left(\theta_{\mathrm{M}}, \infty\right)$ are defined as having less, similar, and more motion during $\mathrm{MC}$ scan, respectively. Similarly, the pairs with $N_{\mathrm{PA}}$ difference in the ranges of $\left(-\infty,-\theta_{\mathrm{N}}\right),\left[-\theta_{\mathrm{N}}, \theta_{\mathrm{N}}\right]$, and $\left(\theta_{\mathrm{N}}, \infty\right)$ are defining as having decreased, similar, and increased $\mathrm{N}_{\mathrm{PA}}$ during $\mathrm{MC}$. In this study, 
the motion score threshold $\theta_{\mathrm{M}}$ was set to $0.4 \mathrm{~mm}$ which is roughly equal to twice the sum of root mean squares of residual standard deviations of the rotational and translational parameters as defined in our previous study [17]. The $N_{\mathrm{PA}}$ threshold $\theta_{\mathrm{N}}$ is chosen as 2 which is twice the average number of false positive PA clusters in testing the performance PA segmentation with M2EDN plus WM masks in Exp 1. Binomial tests were performed to evaluate whether the differences between the numbers of pairs with increased and decreased $\mathrm{N}_{\mathrm{PA}}$ were significant.

To evaluate whether replacement with reacquired data further improved image quality, signed rank tests were performed to compare $\mathrm{N}_{\mathrm{PA}}$ between images with and without replacement. Spearman's correlation coefficients were calculated between the fraction of replaced data and the relative $\mathrm{N}_{\mathrm{PA}}$ changes $\left(\Delta \mathrm{N}_{\mathrm{PA}}\right)$ between images with and without data replacement. Relative $\Delta \mathrm{N}_{\mathrm{PA}}$ (in units of $\%)$ is defined as $200\left(N_{\mathrm{PA}, \mathrm{w}}-N_{\mathrm{PA}, \mathrm{wo}}\right) /\left(N_{\mathrm{PA}, \mathrm{w}}+N_{\mathrm{PA}, \mathrm{wo}}\right)$, where the subscripts $w$ and wo denote with and without replacement, respectively. For all statistical tests, p values less than 0.05 are considered significant.

\subsection{Code and data availability}

All images and the trained CNN models will be shared upon publication of the manuscript.

\section{RESULTS}

Table 1 consists of SEN, PPV, and DSC values for the 4 ROIs in our U-NET segmentation. SEN values are highest for thalamus (0.82) and lowest for midbrain (0.68). PPV values across all 4 ROIs range between $0.8-0.89$. DSC values range from 0.74 to 0.85 across the 4 ROIs, indicating high accuracy of our U-NET model in its segmentation of the 4 ROIs. Figure 2 shows representative $\mathrm{T} 2 \mathrm{w}$ image slices that intersect the four brain regions and the overlaying ROIs generated by the trained U-NET model, demonstrating accurate masks for all ROIs.

Figure 3A and 3B show representative PC MRI images and overlaying PA masks, respectively, generated by the M2EDN. Two false PAs were detected in a sulcus of the brain, as denoted by the arrows, which can be removed by applying the WM mask as shown in Fig. 3C. Figure 4 compares various PA segmentation methods side by side. The mask produced by the M2EDN model located four penetrating arteries that the threshold method was unable to identify, as indicated by the 4 green circles. However, the M2EDN model also identifies one false PA as labeled by the blue circle. 
In Table 2, we assess the performance of the M2EDN model vs. threshold method using the SEN, PPV, and DSC parameters. SEN, PPV, and DSC values are in a lower range for voxel level than for cluster level. Applying WM (denoted as M2EDN+WM) had no effect on SEN as expected but significantly increased both PPV $\left(\mathrm{p}=2.4 \times 10^{-8}\right.$ for both voxel and cluster levels) and DSC ( $\mathrm{p}=2.4 \times 10^{-8}$ for both). When comparing to threshold+WM, M2EDN+WM had higher SEN $\left(\mathrm{p}=4.8 \times 10^{-9}\right.$ (voxel levels) and $1.2 \times 10^{-9}$ (cluster level) ) and DSC $\left(\mathrm{p}=1.6 \times 10^{-8}\right.$ and $1.3 \times 10^{-}$ ${ }^{9}$ ), but lower PPV $\left(\mathrm{p}=6.7 \times 10^{-7}\right.$ and $\left.5.8 \times 10^{-3}\right)$. Importantly, DSC values of M2EDN+WM reached a high value of 0.85 versus only 0.71 of threshold+WM at the cluster level.

Figure 5(A) and (B) show phase and magnitude images acquired with prospective MC and with the conventional PC MRI without the FatNav module, respectively, when there was no head motion in both scans. There is no clear difference between the two sets of images, demonstrating that the FatNav module did not negatively affect image quality.

Table 3 shows a contingency table of cases with various ranges of motion score and $\mathrm{N}_{\mathrm{PA}}$ differences between MC and NoMC scans. Among the 15 cases where MC and NoMC scans had similar motion scores (second column), MC images showed more $\mathrm{N}_{\mathrm{PA}}$ in 9 cases $(60 \%)$ and less $\mathrm{N}_{\mathrm{PA}}$ in $1(7 \%)$ case ( $\mathrm{p}=0.011$; binomial test). Images for one of the former 9 cases are shown in Figure 6, where PAs can be more clearly visualized on the images with MC but appeared blurry on images with NoMC. As a result, the PAs enclosed by the green circles were only segmented by M2EDN in the MC images. The head motion parameters were $M_{\mathrm{T}}=0.79$ $\mathrm{mm}(\mathrm{MC})$ vs $0.59 \mathrm{~mm}(\mathrm{NoMC})$ and $M_{\mathrm{R}}=0.53^{\circ}(\mathrm{MC})$ vs $0.54^{\circ}(\mathrm{NoMC})$. Motion traces for the two scans can be found in Supporting Information Fig. S2. The motion scores for all the 9 cases are between $0.64 \mathrm{~mm}$ and $1.60 \mathrm{~mm}$. In the latter single case where less $\mathrm{N}_{\mathrm{PA}}$ was obtained with $\mathrm{MC}$, the motion ranges were small (score $=0.56 \mathrm{~mm}$ vs $0.64 \mathrm{~mm}$ ). However, small motion range did not always cause smaller $\mathrm{N}_{\mathrm{PA}}$ when $\mathrm{MC}$ is applied, as the two other $\mathrm{MC}$ scans in the same subject also had small motion (score $=0.49 \mathrm{~mm}$ and $0.51 \mathrm{~mm}$ ) but similar $\mathrm{N}_{\mathrm{PA}}$ as the NoMC scan.

The third column in Table 3 shows four cases where the MC scans had more motion than the NoMC scans. MC scans had less $\mathrm{N}_{\mathrm{PA}}$ in three (75\%) of the cases, suggesting that MC did not completely eliminate motion artifacts. Those three scans belonged to the same subject, who had the greatest motion (maximum $M_{\mathrm{T}}=1.2 \mathrm{~mm}$ and $M_{\mathrm{R}}=1.2^{\circ}$ ) during $\mathrm{MC}$ scans and the greatest 
differences in motion score between the MC and NoMC scans, which equaled to $1.57 \mathrm{~mm}, 0.83$ $\mathrm{mm}$, and $1.3 \mathrm{~mm}$, respectively. In the remaining case, $\mathrm{N}_{\mathrm{PA}}$ was similar between $\mathrm{MC}$ and NoMC scans and the motion score difference was only $0.47 \mathrm{~mm}$. Due to the small number of cases, the difference between cases with less and more $\mathrm{N}_{\mathrm{PA}}$ (3 vs 0$)$ was not significant $(\mathrm{p}=0.125$; binomial test). The fourth column shows the distribution of 7 cases where there was less motion during MC than NoMC scans. More and similar $\mathrm{N}_{\mathrm{PA}}$ were obtained with $\mathrm{MC}$ in 6 and 1 cases, respectively. The difference between cases with more and less $\mathrm{N}_{\mathrm{PA}}(6 \mathrm{vs} 0)$ was significant ( $\mathrm{p}=$ 0.016; binomial test). The list of all $M_{\mathrm{T}}, M_{\mathrm{R}}, \mathrm{N}_{\mathrm{PA}}$ values for the $\mathrm{MC}$ and NoMC scans can be found on the Supporting Information Table S2.

Figures 7 shows relative $\Delta \mathrm{N}_{\mathrm{PA}}$ versus the fraction of data replaced by reacquired data. No significant correlation ( $\rho=0.06 ; p=0.64$ ) was observed and signed rank test between $N_{P A}$ values with and without replacement gave a $\mathrm{p}$ value of 0.11 , suggesting no significant improvement in PA visualization with data reacquisition.

\section{DISCUSSION}

In this study, we developed FatNav based prospective MC method for improving PA visualization in 2D PC MRI sequence and evaluated its performance using automatic PA segmentation methods based on M2EDN and 3D U-NET. Compared to the NoMC scans with similar motion scores, MC significantly improved the visualization of PA and increased the number of segmented PAs. However, we found no further improvement by reacquiring data affected by head motion. The convolutional neural networks increased the SEN and DSC of PA delineation compared to conventional threshold-based method.

Although navigator based prospective MC has been incorporated into various MRI sequences [22-29], it has never been applied to PC MRI. In previous applications, a complete dataset of navigator echo or image were acquired in a single time block. This is impractical for PC MRI due to its short repetition time. Therefore, the k-space data for navigator and main images were acquired in an interleaved fashion, which increased the possibility of artifacts due to the introduction of additional gradient pulses between RF excitations for water signal. However, no visible artifacts were introduced, as illustrated in Fig. 2, which might be explained by the nulling of the zeroth order gradient moment of the FatNav module. A second distinction from previous 
studies is that the fat signal was used for navigators which avoids reducing the water signal and thus image intensity of the main image.

A shortcoming of the interleaved acquisition is that it decreased the frequency for motion parameter updates, making it challenging to measure and correct sudden motions. We tried to ameliorate this issue by reacquiring data blocks during which motion had occurred. More strict criteria were applied to detect motion than in previous studies by requiring no relative motion between three consecutive FatNav images. The rationale behind the strict criteria is that sudden motion can lead to artifacts in FatNav images which might manifest as changes in motion parameters during image registration. The criteria should be sufficient to detect stepwise motion but its sensitivity for detecting spike-like motion remains unclear.

While improved PA visualization and $\mathrm{N}_{\mathrm{PA}}$ were observed in scans with MC compared to NoMC, we did not find further improvement with data reacquisition, in apparent contradiction to previous studies where data reacquisition was found to be important for complete correction of image artifacts $[22,25]$. There are several possible reasons for the lack of improvement in our study. First, in contrast to the correction of deliberate large motions in the previous studies [22, 25], our study focused on real-life scanning conditions in which subjects were not asked to make deliberate large head movements, but to keep as still as possible. As a result, decent image quality was obtained in most cases even without data reacquisition, leaving less room for further improvement with data reacquisition. Second, as discussed above, sudden spike-like motions may not be detected by FatNav image registration. Third, although $\sim 10-90 \%$ data blocks were replaced by reacquired data using our criteria, some of them may not truly represent data with more motion due to the intrinsic measurement errors in $M_{T}$ and $M_{R}$ and the small scores of the true motion. A related issue is the slight dissimilarity between the FatNav images and the reference FatNav image acquired during the TSE scans which not only caused systematic bias but might also increase random errors in the estimated motion parameters.

We conducted CNN-based PA and WM segmentation to determine whether the combination of these two masks can provide more accurate segmentation results than the existing threshold based method. According to the data we gathered, the new method identified a greater number of PAs than the previous threshold method. In Table 1, it is evident that the CNN-based method 
improved overall accuracy of PA identification. PPV values were lower for both M2EDN and M2EDN+WM and SEN values were higher compared to those of threshold+WM. A lower PPV value indicates a higher false positive rate and this is exhibited in Figure 3C as the M2EDN mask identifies one false artery that the threshold method did not identify. SEN values for the M2EDN masks are much higher than the threshold method indicating fewer false negatives. Adding the WM mask on top of the PA segmentation obtained slightly greater DSC values as opposed to M2EDN alone, suggesting that the WM mask was effective in excluding false PAs located on the cortical surface.

Although CNN-based tissue segmentation of $7 \mathrm{~T}$ healthy brain MRI images has been reported before $[30,31]$, our study is the first to evaluate the performance of such an approach for WM segmentation based on T2w images. CEREBRUM-7T employed 3 layers of 3D convolutional blocks and achieved DSC of 0.9 and 0.86 in WM and BG [30], respectively, which are superior to the DSC results in Table 1 . However, $\mathrm{T}_{1}$ weighted images which have higher tissue contrast were employed. Khandelwal et. al. segmented gray matter in $7 \mathrm{~T}$ ex vivo T2w images with $0.3 \times 0.3 \times 0.3 \mathrm{~mm} 3$ resolution and achieved DSC of $78.5 \%$ to $98.5 \%$. However, the WM was not segmented [31].

There are several proposed causes of SVD. One in particular is the occlusion of PAs which can result in a lacunar infarct downstream of the occlusion. On PC MRI images, occluded arteries without blood flow will not appear on the scan. Fewer identified arteries in our segmentations could be an indicator of potential diseased PAs. Longitudinal studies could assess how other factors such as cholesterol level, blood pressure, or blood glucose levels affect the blood flow through PAs. Observing whether blood flow improves or worsens with therapeutic interventions could be useful for developing effective prevention and treatment strategies for SVD.

Our study has several limitations. First, due to different sequence parameters for FatNav between T2w and PC MRI scans, there existed a systematic error in the motion parameters and as a result the first repetition of PC MRI had to been discarded. In the future, more accurate image registration algorithm should be developed and incorporated into the sequence. Second, in evaluating the performance of MC, controlled deliberate large motion was typically performed. However, we did not perform such comparison, but instead based our evaluation on real-life 
data, in which the motion is only $\leq 1.2 \mathrm{~mm}$ and $1.2 \mathrm{deg}$. In the subject with the largest motion (Subject 2 in Table S2 and column 3 row 2 in Table 3), MC did not completely overcome the detrimental effects of motion and had less PAs than the NoMC images which had much less motion. However, more cases are needed to further test whether MC can still be effective in cases with large motion (motion score $\sim$ or $>1 \mathrm{~mm}$ ). Third, due to the relative long TR to acquire the complete k-space data for each FatNav image, spike like motion may not be detected. Other motion detection approaches such as that based on free induction decay signal should be explored in future development [31], which may enable further improvement in image quality by data reacquisition.

\section{CONCLUSIONS}

We have developed a prospective motion correction method for PC MRI which improved the visualization of PA and segmented PA counts in centrum semiovale at 7 T. Furthermore, we have improved the overall accuracy (DSC) and sensitivity of PA segmentation using CNN-base approaches. The developed PA imaging and segmentation methods may help illuminate the mechanisms of pathophysiological changes of PAs in SVD.

\section{ACKNOWLEDGEMENTS}

The project described was partly supported by United States National Institutes of Health through grant 5R21NS095027-02 and the National Center for Advancing Translational Sciences (NCATS), National Institutes of Health, through Grant Award Number UL1TR002489.

\section{REFERENCES}

1. Wardlaw, J.M., Small vessel disease: mechanisms and clinical implications. Lancet, 2019.

2. Caplan, L.R., Lacunar infarction and small vessel disease:pathology and pathophysiology. National Library of Medicine, 2015.

3. Low, A., et al., Inflammation and cerebral small vessel disease: A systematic review. Ageing Research Reviews, 2019. 53: p. 100916.

4. van der Flier, W.M., et al., Small vessel disease and general cognitive function in nondisabled elderly: the LADIS study. Stroke, 2005. 36(10): p. 2116-20.

5. Kalaria, R.N. and T. Erkinjuntti, Small vessel disease and subcortical vascular dementia. J Clin Neurol, 2006. 2(1): p. 1-11.

6. Kisler, K., et al., Cerebral blood flow regulation and neurovascular dysfunction in Alzheimer disease. Nat Rev Neurosci, 2017. 
7. Herrmann, L.L., M. Le Masurier, and K.P. Ebmeier, White matter hyperintensities in late life depression: a systematic review. J Neurol Neurosurg Psychiatry, 2008. 79(6): p. 61924.

8. de Laat, K.F., et al., Loss of white matter integrity is associated with gait disorders in cerebral small vessel disease. Brain, 2011. 134(Pt 1): p. 73-83.

9. Mestre, H., et al., Perivascular spaces, glymphatic dysfunction, and small vessel disease. Clin Sci (Lond), 2017. 131(17): p. 2257-2274.

10. Zong, X. and W. Lin, Quantitative phase contrast MRI of penetrating arteries in centrum semiovale at 7T. Neurolmage, 2019. 195: p. 463-474.

11. Geurts, L., et al., Better and faster velocity pulsatility assessment in cerebral white matter perforating arteries with 7T quantitative flow MRI through improved slice profile, acquisition scheme, and postprocessing. Magn Reson Med, 2018. 79(3): p. 1473-1482.

12. Gallichan, D., J.P. Marques, and R. Gruetter, Retrospective correction of involuntary microscopic head movement using highly accelerated fat image navigators (3D FatNavs) at 7T. Magn Reson Med, 2016. 75(3): p. 1030-9.

13. Lian, C., et al., Multi-channel multi-scale fully convolutional network for $3 D$ perivascular spaces segmentation in 7T MR images. Med Image Anal, 2018. 46: p. 106-117.

14. Ronneberger, O., P. Fischer, and T. Brox, U-Net: Convolutional Networks for Biomedical Image Segmentation. MICCAI, 2015. 9351: p. 234-241.

15. Zong, X., et al., Visualization of perivascular spaces in the human brain at 7T: sequence optimization and morphology characterization. Neuroimage, 2016. 125: p. 895-902.

16. Busse, R.F., et al., Fast spin echo sequences with very long echo trains: design of variable refocusing flip angle schedules and generation of clinical T2 contrast. Magn Reson Med, 2006. 55(5): p. 1030-7.

17. Zong, $\mathrm{X}$., et al., Effects of motion and retrospective motion correction on the visualization and quantification of perivascular spaces in ultrahigh resolution T2-weighted images at 7T. Magn Reson Med, 2021.

18. Brownlee, J., Gentle Introdcution to Pooling Layers for Convolutional Neural Networks. 2019.

19. Zong, X., et al., Morphology of perivascular spaces and enclosed blood vessels in young to middle-aged healthy adults at 7T: Dependences on age, brain region, and breathing gas. Neurolmage, 2020. 218: p. 116978.

20. Zhang, J., UNet-Line by Line Explanation. 2019.

21. Griswold, M.A., et al., Generalized autocalibrating partially parallel acquisitions (GRAPPA). Magn Reson Med, 2002. 47(6): p. 1202-10.

22. Tisdall, M.D., et al., Volumetric navigators for prospective motion correction and selective reacquisition in neuroanatomical MRI. Magn Reson Med, 2012. 68(2): p. 38999.

23. Lee, C.C., et al., A prospective approach to correct for inter-image head rotation in fMRI. Magn Reson Med, 1998. 39(2): p. 234-43.

24. White, N., et al., PROMO: Real-time prospective motion correction in MRI using imagebased tracking. Magn Reson Med, 2010. 63(1): p. 91-105. 
25. Frost, R., et al., Prospective motion correction and selective reacquisition using volumetric navigators for vessel-encoded arterial spin labeling dynamic angiography. Magn Reson Med, 2016. 76(5): p. 1420-1430.

26. Hoinkiss, D.C. and D.A. Porter, Prospective motion correction in $2 D$ multishot MRI using EPI navigators and multislice-to-volume image registration. Magn Reson Med, 2017. 78(6): p. 2127-2135.

27. Mattern, H., et al., Prospective motion correction improves high-resolution quantitative susceptibility mapping at 7T. Magn Reson Med, 2019. 81(3): p. 1605-1619.

28. Mattern, H., et al., Prospective motion correction enables highest resolution time-offlight angiography at 7T. Magn Reson Med, 2018. 80(1): p. 248-258.

29. Simegn, G.L., et al., Real-time simultaneous shim and motion measurement and correction in glycoCEST MRI using double volumetric navigators (DvNavs). Magn Reson Med, 2018.

30. Svanera, M., et al., CEREBRUM-7T: Fast and Fully Volumetric Brain Segmentation of 7 Tesla MR Volumes. Hum Brain Mapp, 2021. 42(17): p. 5563-5580.

31. Khandelwal, P., et al., Gray Matter Segmentation in Ultra High Resolution 7 Tesla ex vivo T2w MRI of Human Brain Hemispheres. arXiv, 2021. 2110.07711.

\section{FIGURE CAPTIONS}

Figure 1: Pulse sequence of the phase contrast-MRI sequence with FatNav-based motion correction.

Figure 2: (A) Representative T2w images in a single subject. (B) Overlaid tissue segmentation masks. The yellow, green, red, and blue regions are $\mathrm{WM}, \mathrm{BG}, \mathrm{TH}$, and $\mathrm{MB}$, respectively.

Figure 3: (A) Phase and magnitude images of a representative subject. (B) PA masks generated from the M2EDN network shown as red dots. (C) Resampled WM mask generated from the UNET shown in yellow. The arrows denote two false positive clusters on the cortical surface.

Figure 4: (A) Phase and magnitude images in a representative subject. (B) PA masks generated from the thresholding method, as shown in red. (C) PA masks generated by the M2EDN network as shown in red. (D) the ground truth mask as shown in red. In $(A)-(C)$, the green circles denote true PA clusters that were only detected using M2EDN. The blue circle denotes a false PA cluster in the M2EDN mask that is absent in the threshold mask.

Figure 5: Comparison of PC-MRI images acquired (A) with and (B) without the fat navigator module. The real-time feedback was also turned on in $(\mathrm{A})$.

Figure 6: Comparison of (A) magnitude and (B) phase contrast images with MC off (left panel) and on (right panel). The green circles enclose PAs that were only segmented by M2EDN in the MC but not NoMC images.

Figure 7: Scatter plots of relative $\Delta \mathrm{N}_{\mathrm{PA}}$ versus the fraction of data replaced by those with smaller motion scores. The red line is a least square fit.

Supporting Information Figure S1: The architecture of the 3D U-NET for brain tissue segmentation. The numbers above the rectangles specify the input/output channels of different layers, while the numbers above the pink arrows specify the dropout rate of the Dropout layer. 
Supporting Information Figure S2: Motion parameter traces during the (A) NoMC and (B) MC scans in Fig. 6, showing similar degrees of motion during the MC on and off scans. Different colors represent different motion directions or rotation axes.

\section{TABLE CAPTIONS}

Table 1: SEN, PPV, and DSC of TH, BG, MB, and WM masks obtained using UNET segmentation of $\mathrm{T} 2 \mathrm{w}$ images.

Table 2: SEN, PPV, and DSC of penetrating artery masks obtained using the M2EDN, $\mathrm{M} 2 \mathrm{EDN}+\mathrm{WM}$, and threshold+WM methods. The numbers 61 and 50 inside the parentheses of the second column correspond to all images and those with WM masks, respectively, in Exp. 1. The \# and + signs denote significant difference from the corresponding M2EDN and threshold+WM results, respectively.

Table 3: The contingency table of cases with different motion score and $\mathrm{N}_{\mathrm{PA}}$ relationships between a MC scan and the NoMC scan in the same subject. The labels refer to MC relative to NoMC. The - and + signs in the parentheses denote decline and improvement, respectively, in PA visibility due to MC, as compared to NoMC.

Supporting Information Table S1: MRI parameters for the MPRAGE, VFA-TSE, and MP2RAGE sequences. In the PC sequence, VENC $=4 \mathrm{~cm} / \mathrm{s}$ and a one-sided flow encoding were employed, thereby the flow encoding gradient was turned on and off alternatively in different TRs.

Supporting Information Table S2: list of the subject ID, NPA, MT, MR, and MT+MR difference for all 26 image pairs grouped according to the NPA and motion range differences between MC and NoMC scans. 
(A)

(B)
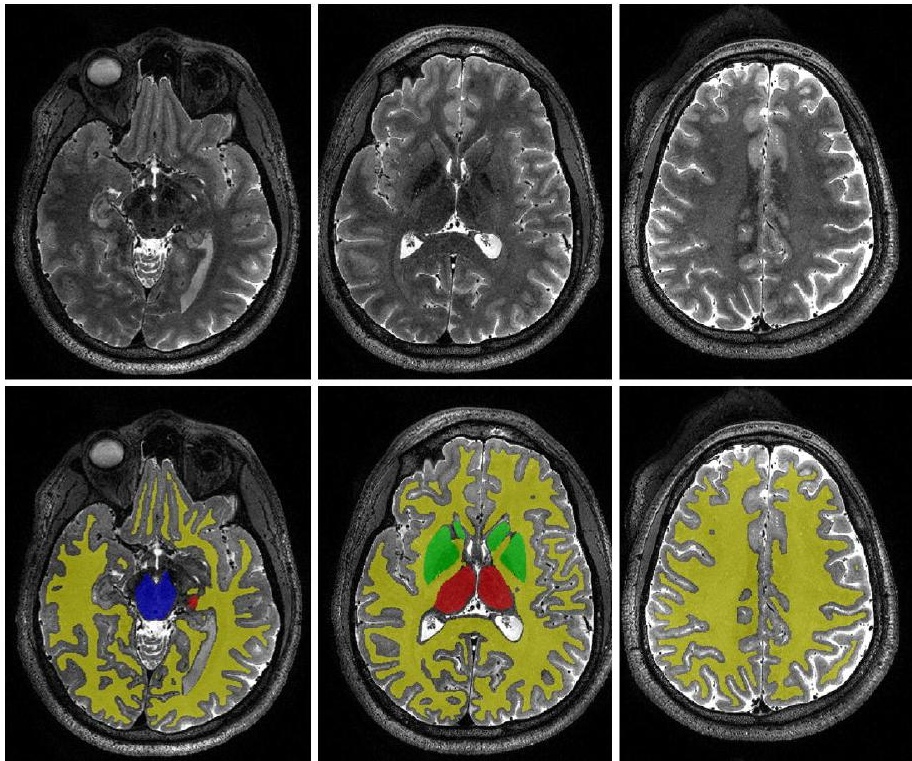
(A)

Phase

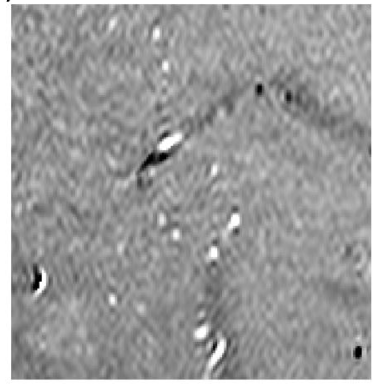

Magnitude

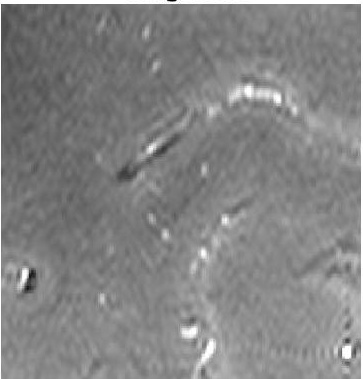

(B)

Phase

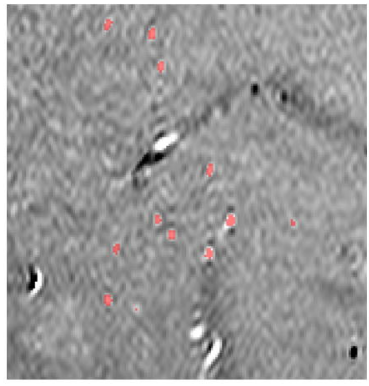

Magnitude

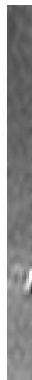

(C)

Phase

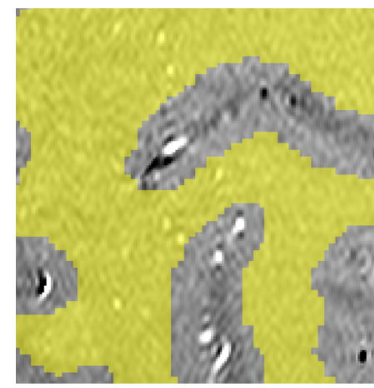

\section{Magnitude}

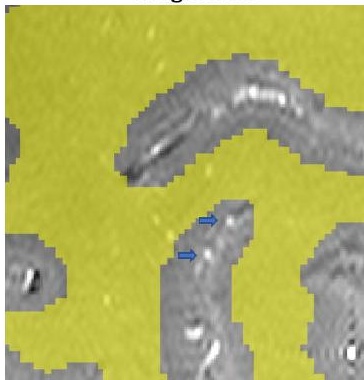




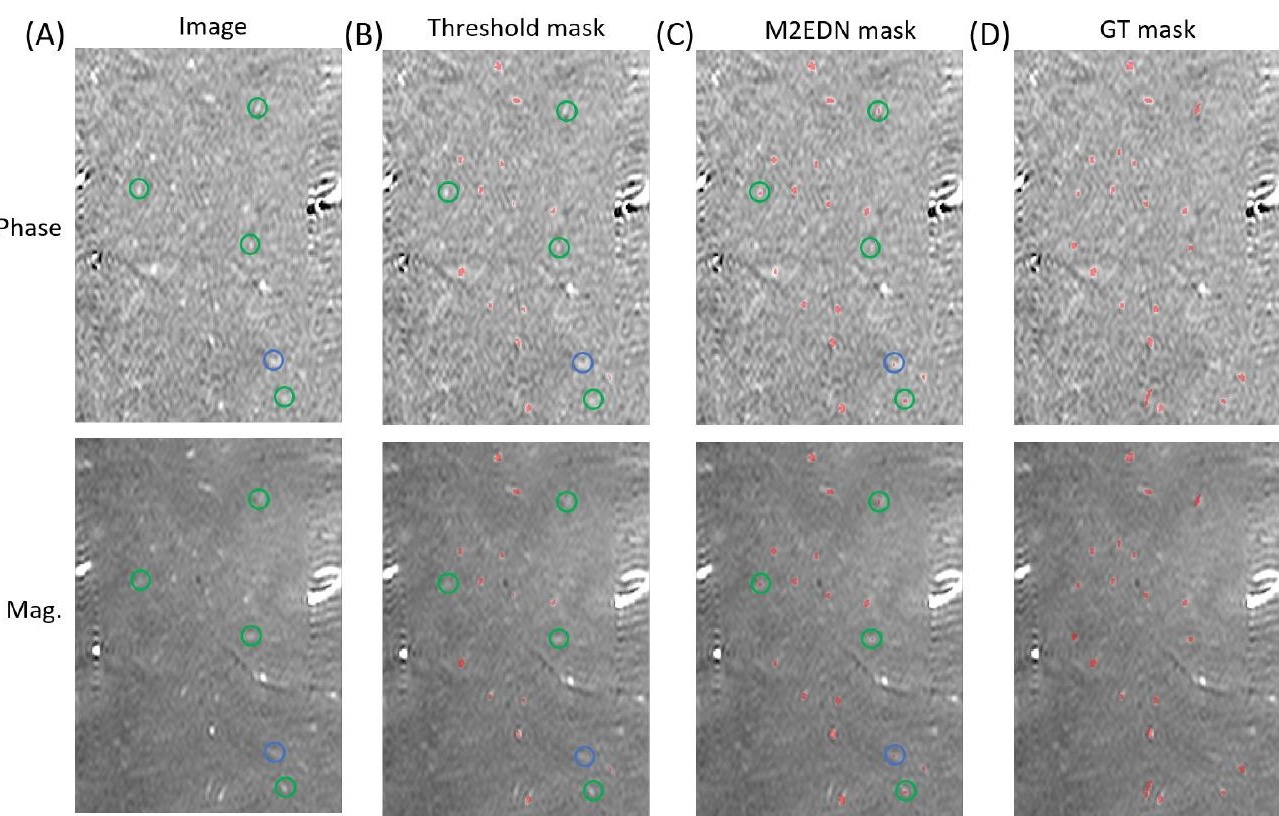


(A)

\section{FatNav/MC on}

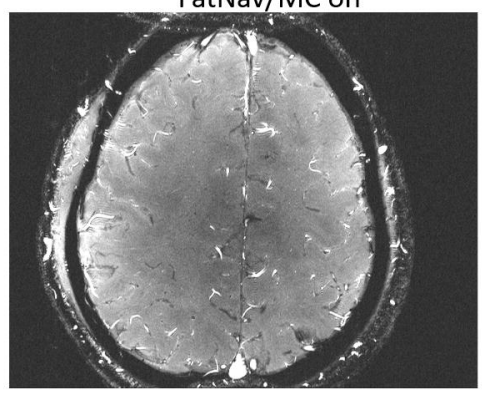

Mag

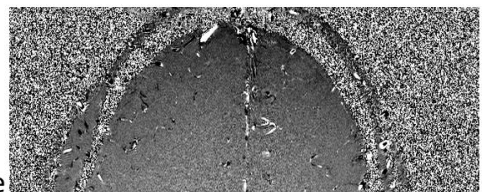

Phase

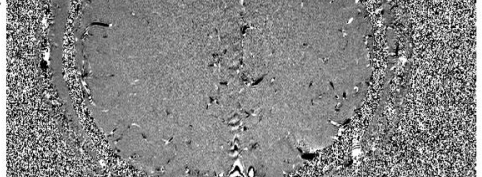
17. F.
(B)

\section{FatNav/MC off}
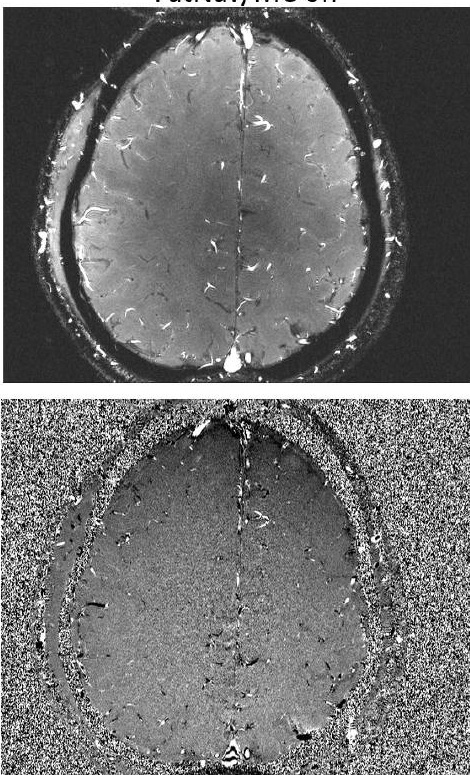


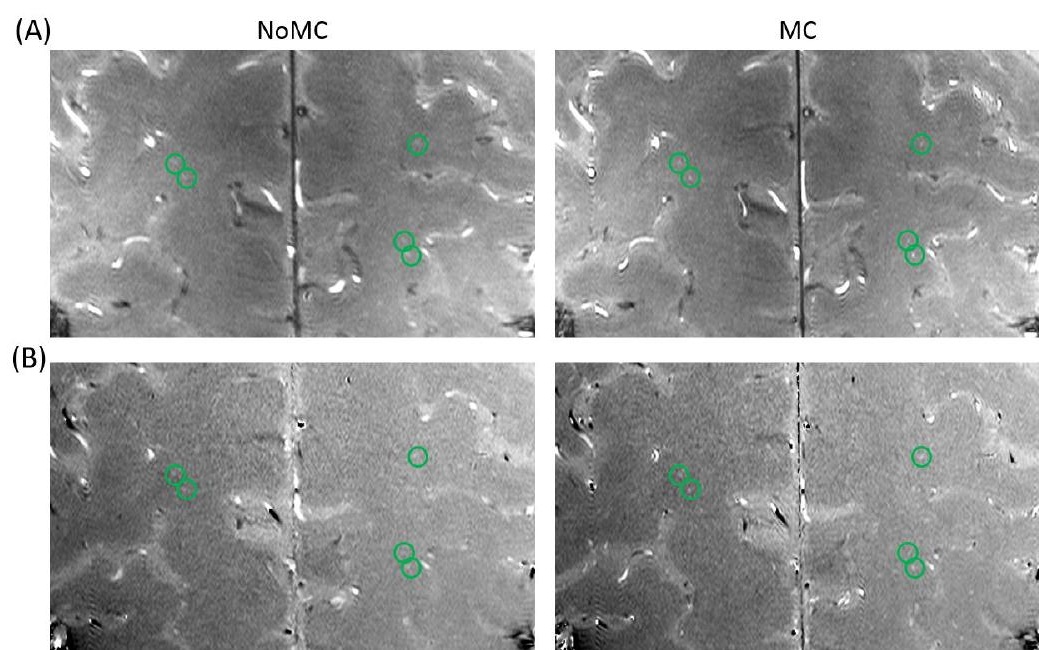




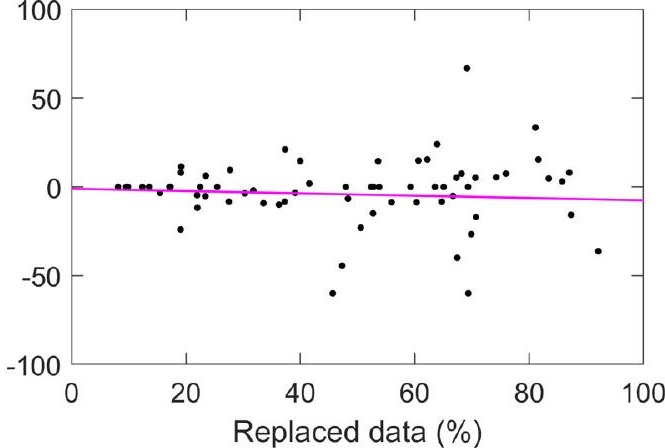

